\title{
SOME PROPERTIES OF THE SIGNATURE OF COMPLETE INTERSECTIONS
}

\section{A. LIBGOBER}

ABstract. We prove that:

(1) The signature of complete intersections is a monotone function of degrees of defining equations.

(2) The signature of $n$-dimensional complete intersection (except for $\mathbf{C P}^{n}$ ) is positive for $n \equiv 0(\bmod 4)$ and is negative for $n \equiv 2(\bmod 4)$.

The results of this note are motivated by the following question originally asked by R. Kulkarni and J. Wood. What is the sign of the signature of even-dimensional nonsingular algebraic hypersurfaces? Here we prove the following

THEOREM 1. Let $n$ be even and $\tau_{n}\left(a_{1}, \ldots, a_{r}\right)$ denote the signature of a nonsingular $n$-dimensional complete intersection of hypersurfaces of degrees $a_{1}, \ldots, a_{r}$ in $\mathbf{C P}^{n+r}$. Then $\tau_{n}\left(a_{1}, \ldots, a_{r}\right)$ is a monotone function of each argument (increasing for $n \equiv 0$ $(\bmod 4)$ and decreasing for $n \equiv 2(\bmod 4))$.

Recall that all nonsingular complete intersections of given dimension $n$ and multidegree $\left(a_{1}, \ldots, a_{r}\right)$ are diffeomorphic. Therefore $\tau_{n}\left(a_{1}, \ldots, a_{r}\right)$ is a correctly defined function.

In particular we have

THEOREM 2. $(-1)^{n / 2} \tau_{n}\left(a_{1}, \ldots, a_{r}\right)>0$ except for the cases $a_{i}=1(i=1, \ldots, r)$, when the signature is 1 , or $n \equiv 2 \bmod 4, a_{1}=2, a_{i}=1(i=2, \ldots, r)$ when the signature is zero.

Note that Theorem 2 is also proved by J. Wood [2]. We suggest our proof for its simplicity.

Lemma. Suppose that $a_{i} \neq 1$. Then

$$
\begin{aligned}
\tau_{n}\left(a_{1}, \ldots, a_{r}\right)= & \tau_{n}\left(a_{1}, \ldots, a_{i-1}, a_{i+1}, \ldots, a_{r}\right) \\
& +\tau_{n}\left(a_{1}, \ldots, a_{i-1}, a_{i}-1, a_{i+1}, \ldots, a_{r}\right) \\
& -\tau_{n-2}\left(a_{1}, \ldots, a_{i-1}, a_{i}-1, a_{i}, a_{i+1}, \ldots, a_{r}\right) .
\end{aligned}
$$

Proof. Simple arguments of Bertini theorem type show that we may choose hypersurfaces $H^{a_{1}}, \ldots, H^{a_{r}}$ of degrees $a_{1}, \ldots, a_{r}$ respectively, a hypersurface $\bar{H}^{a_{i}-1}$ of degree $a_{i}-1$ and hyperplane $L$ in such a way that the following varieties are nonsingular:

Received by the editors March 14, 1978 and, in revised form, November 1, 1978.

AMS (MOS) subject classifications (1970). Primary 14M10, 57A15, 57D99. 
(a) $V_{n}\left(a_{1}, \ldots, a_{r}\right)=H^{a_{1}} \cap \cdots \cap H^{a^{a}}$.

(b) $\underline{\underline{V}}_{n}=H^{a_{1}} \cap \cdots \cap H^{a_{1-1}} \cap L \cap H^{a_{1+1}} \cap \cdots \cap H^{a_{1}}$.

(c) $\bar{V}_{n}=H^{a_{1}} \cap \cdots \cap H^{a_{1-1}} \cap \bar{H}^{a_{1}-1} \cap H^{a_{1+1}} \cap \cdots \cap H^{a}$.

(d) $S=V_{n}\left(a_{1}, \ldots, a_{r}\right) \cap \bar{H}^{a_{1}-1} \cap L$.

Let $h$ denote the canonical generator of $H^{2}\left(\mathrm{CP}^{n+r}, \mathrm{Z}\right)$. Then the signatures of $V_{n}, \bar{V}_{n}, \overline{\bar{V}}_{n}, S$ are equal to the virtual indices of the sets of cohomology classes

$$
\begin{gathered}
\left(a_{1} h, \ldots, a_{r} h\right),\left(a_{1} h, \ldots, a_{i-1} h, h, a_{i+1} h, \ldots, a_{r} h\right), \\
\left(a_{1} h, \ldots, a_{i-1} h,\left(a_{i}-1\right) h, a_{i+1} h, \ldots, a_{r} h\right),\left(a_{1} h, \ldots, a_{i} h, \ldots, a_{r} h,\left(a_{i}-1\right) h, h\right)
\end{gathered}
$$

respectively. Therefore the statement of the lemma is a particular case of the recursive equation for virtual index [1, Theorem 9.3.1].

Now we are ready to conclude the proof of Theorems 1 and 2.

Proof of TheOREM 2. We proceed by induction by dimension, degree and number of nonlinear defining equations of complete intersection. The case of dimension zero is trivial because the signature is just the degree (i.e., the number of points). Suppose that the number of defining equations is one and Theorem 2 holds for all dimensions less than $n$. If $a_{1}=2$ then $\tau_{n}\left(V_{n}(2)\right)=1+(-1)^{n / 2}$. Indeed, rk $H_{n}\left(V_{n}(2), \mathbf{R}\right)=2$. As the basis one can choose the homology class of the section of $V_{n}(2)$ by $\mathrm{CP}^{(n / 2)+1}$ for which $h^{2}=\operatorname{deg} V_{n}(2)=2$, and vanishing cycle $c$ (i.e., $(h, c)=0)$ for which $(c, c)=(-1)^{n / 2} \cdot 2$. Hence if $n \equiv 0(\bmod 4)$ we obtain that $\tau_{n}\left(V_{n}(3)\right)>3$ from the lemma and inductive assumptions, because $\tau\left(V_{n}(3)\right)=$ $\tau\left(V_{n}(2)\right)+\tau_{n}\left(V_{n}(1)\right)-\tau\left(V_{n-2}(2,3)\right)$. If $n \equiv 2(\bmod 4)$ we have $\tau\left(V_{n-2}(2,3)\right)<$ -3 because

$$
\tau\left(V_{n-2}(2,3)\right)=\tau\left(V_{n-2}(1,3)\right)+\tau\left(V_{n-2}(1,3)\right)-\tau\left(V_{n-4}(2,3)\right) .
$$

Therefore we obtain the inequality $(-1)^{n / 2} \tau\left(V_{n}\left(a_{1}\right)\right)>0$ for every $a_{1}$. The assertion of Theorem 2 follows now by induction for arbitrary $r$ from the equation of the lemma.

Proof of TheOREM 1. By Theorem 2 we have

$$
\begin{aligned}
& (-1)^{n / 2}\left[\tau\left(V_{n}\left(a_{1}, \ldots, a_{i-1}, 1, a_{i+1}, \ldots, a_{r}\right)\right)\right. \\
& \left.-\tau\left(V_{n-2}\left(a_{1}, \ldots, a_{i-1}, 1, a_{i}-1, a_{i}, \ldots, a_{r}\right)\right)\right]>0
\end{aligned}
$$

Hence by the lemma

$$
(-1)^{n / 2}\left[\tau\left(V_{n}\left(a_{1}, \ldots, a_{r}\right)\right)-\tau\left(V_{n}\left(a_{1}, \ldots, a_{i-1}, a_{i}-1, a_{i+1}, \ldots, a_{r}\right)\right)\right]>0
$$

i.e., $\tau$ is monotone in the appropriate sense as a function of $a_{i}$.

REMARK. For a detailed investigation of the topology of even dimensional hypersurfaces and complete intersections [2] one needs the following inequality:

$$
(-1)^{n / 2} \tau_{n}\left(a_{1}, \ldots, a_{r}\right)>5 \text {. }
$$

It holds for arbitrary $n, a_{1}, \ldots, a_{r}$ except for the following cases:

(i) $a_{1}=\cdots=a_{r}, n$ arbitrary, then $\tau\left(V_{n}(1, \ldots, 1)\right)=1$.

(ii) $a_{1}=2, a_{2}=\cdots=a_{r}=1, n$ arbitrary. Then $\tau\left(V_{n}(2)\right)=1+(-1)^{n / 2}$.

(iii) $a_{1}=2, a_{2}=2, n=2$. Then $\tau\left(V_{2}(2,2)\right)=-4$. This follows by repeated use of the lemma. 
I am grateful to B. Moishezon and D. Randell for helpful remarks.

\section{REFERENCES}

1. F. Hirzebruch, Topological methods in algebraic geometry, Springer-Verlag, Berlin and New York, 1966.

2. J. Wood, A connected sum decompasition for complete intersections, Proc. Sympos. Pure Math., vol. 32, part 2, Amer. Math. Soc., Providence, R. I., 1978, pp. 191-193.

School of Mathematics, The Institute for Advanced Study, Prnnceton, New Jersey 08540

Current address: Department of Mathematics, University of Illinois at Chicago Circle, Chicago, Illinois 60680 